\title{
A Wideband Circularly Polarised Cross-Slot Antenna with an L- Shaped Feed-Line
}

\author{
Mostafa G. Aly, Yi Wang \\ Department of Engineering Science, University of Greenwich, Central Avenue, Chatham Maritime, Kent ME4 4TB, UK \\ M.G.Aly@greenwich.ac.uk_Yi.Wang@greenwich.ac.uk
}

Keywords: slot antenna, circularly polarised, axial ratio.

\begin{abstract}
This paper presents a new wideband and circularly polarised (CP) slot antenna realised by using an L-shaped feed underneath the cross-slot antenna, and tapered to the conventional microstrip feed-line. The proposed antenna is fabricated with an area of $75 \times 80 \mathrm{~mm}^{2}$. The simulated results showed that the antenna had an impedance matching bandwidth of $34 \%$ from 1.3 to $1.83 \mathrm{GHz}$, and an axial ratio (AR) bandwidth of $20 \%$ from 1.36 to $1.66 \mathrm{GHz}$. A maximum gain value of $3.4-3.8 \mathrm{dBi}$ has been achieved within the operating band. The proposed antenna is of a simple and single-substrate structure, suitable for the Global Navigation Satellite Systems (GNSS) application. The impedance bandwidth and radiation patterns have been confirmed by measurement.
\end{abstract}

\section{Introduction}

Circular polarisation $(\mathrm{CP})$ feature has grasped a lot of attention in modern mobile wireless communications, radars, radio frequency identifications, and satellite systems such as the Global Navigation Satellite Systems (GNSS) application. The major advantages of $\mathrm{CP}$ antennas are its capability to transmit and receive signals with no restricted orientation [1] and its ability of weather penetration with rejection of multipath signals and thus enhancing the polarisation efficiency of the receiving antenna [2]. CP antennas can be realised using either single or dual-feed structures. Dual-feed configuration is often more complex in implementation than the single-feed, but offers more bandwidth. CP antennas can be of co-planar, microstrip or slot structures and coupled via slots, slits, stubs, and/or the hybrids. The printed inverted-F antenna described in [3] had an axial ratio bandwidth (ARBW) of $3.68 \%$ covering the industrial, scientific and medical (ISM) band. In [4], a simple corner truncated microstrip antenna had a vertical L-shaped ground plane. The air separation between the patch and the substrate helped in achieving an ARBW of $10.4 \%$ for the wireless local area network (WLAN) band. An antipodal Y-shaped strip introduced in [5] had a U-shaped microstrip feed-line and a square slot on the ground plane, managed to record a $41.3 \%$ of ARBW from 4.4 to $6.67 \mathrm{GHz}$. Another method to enhance the impedance BW and ARBW is to use monopole or slot antennas. Slot antennas having an L-shape and fed by a Cshaped feed-line can also generate $\mathrm{CP}$ radiation [6] where
23\% ARBW was reported covering the global positioning systems (GPS) band. In [7] a tilted monopole antenna fed with a microstrip feed-line having a partial ground plane with a triangular slit was presented, offering an overall ARBW of $59.1 \%$ to operate for the GNSS system of Beidou in China. A bi-directional $\mathrm{C}$-shaped monopole antenna was proposed in [8] with an open end loop at the partial ground plane to couple the electromagnetic waves. An ARBW of $65.2 \%$ was obtained serving WLAN. A reflector was also added underneath the substrate to obtain a unidirectional pattern with a higher gain, but at the cost of reduced ARBW.

In this communication, a bi-directional wideband $\mathrm{CP}$ slot antenna is presented for GNSS application from 1.3 to 1.83 $\mathrm{GHz}$. The wideband CP operation is accomplished using an L-shaped metal strip added to the feed-line placed underneath the cross-slot. A considerable impedance and 3-dB AR bandwidths is achieved with a compact overall antenna size and on a single substrate. The proposed antenna is designed and simulated using the CST Microwave Studio simulator. The antenna structure is described in Section 2. Section 3 contains the parametric studies, while simulated and measured results are demonstrated in Section 4. A conclusion is drawn in Section 5.

\section{Antenna Structure}

The proposed structure of the slot antenna is shown in Fig. 1. The antenna is fabricated using a single substrate of Rogers RO4003C with a dielectric constant of 3.55 and thickness of $0.813 \mathrm{~mm}$. The overall size of the antenna is $75 \times 80 \mathrm{~mm}^{2}$. The cross-slot is of $L_{c} \times W_{c}(61 \mathrm{~mm} \times 13.5 \mathrm{~mm})$ etched in the ground plane at the top of the substrate. A supplementary slot is added to the upper vertical half in order to control the ARBW. The slot is fed from the opposite side of the substrate using an L-shaped metal strip of a width $W_{l}$ of $13.5 \mathrm{~mm}$ and lengths $L_{l}$ and $L_{l l}$ of $32 \mathrm{~mm}$ and $10.25 \mathrm{~mm}$ respectively. This is tapered to a $50 \mathrm{ohm}$ microstrip line. The transition structure is optimised for impedance matching. A slot is inserted in the Lshape feed-line for further control of the impedance matching. In order to achieve the $\mathrm{CP}$ operation, the fundamental resonant mode of the cross-slot is splitted into two degenerated modes using the L-shaped feeding strip. By adjusting the length and width of the cross-slot antenna, two orthogonal modes of the same amplitude and in quadrateure phase are achieved. 


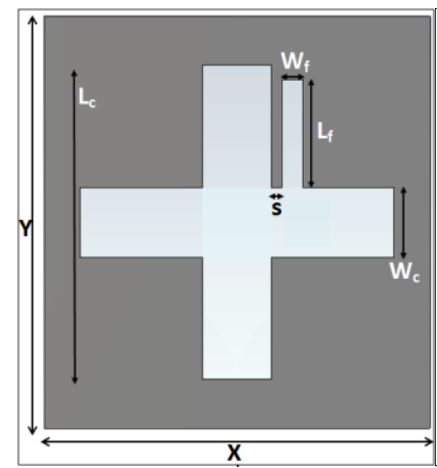

(a)

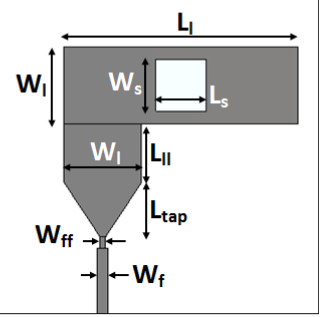

(b)
Figure 1: Geometry of the cross-slot CP antenna: (a) top view (b) bottom view. $\mathrm{X}=75, \mathrm{Y}=80, \mathrm{~L}_{\mathrm{c}}=61, \mathrm{~W}_{\mathrm{c}}=13.5, \mathrm{~W}_{\mathrm{l}}=13.5$, $\mathrm{L}_{\mathrm{l}}=32, \mathrm{~L}_{l l}=10.25, \mathrm{~W}_{\mathrm{f}}=1.85, \mathrm{~W}_{\mathrm{ff}}=1, \mathrm{~L}_{\mathrm{tap}}=10.5, \mathrm{~L}_{\mathrm{s}}=9, \mathrm{~W}_{\mathrm{s}}=9.25$, $\mathrm{L}_{\mathrm{f}}=21, \mathrm{~W}_{\mathrm{f}}=4, \mathrm{~s}=2$ (unit: $\mathrm{mm}$ )

\section{Parametric Study}

To reach the final design in Fig. 1, extensive studies and simulations were carried out to optimize the impedance and AR bandwidths. To attain the impedance matching, it was found that the vertical L-shaped feed-line length $\left(L_{l l}\right)$ was the parameter that had a great effect upon the return loss BW (RLBW) while not affecting the ARBW as can be seen from Fig. 2.

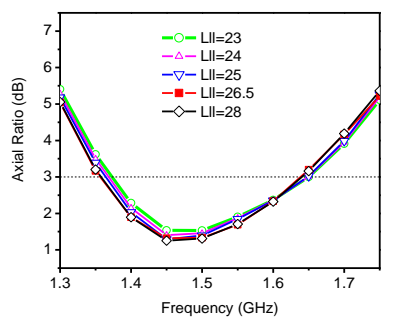

(a)

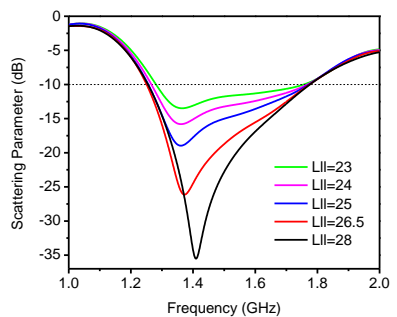

(b)
Figure 2: Effect of vertical L-length $\left(L_{l l}\right)$ on (a) AR (b) $\mathrm{S}_{11}$

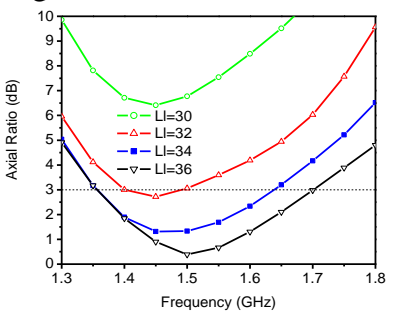

(a)

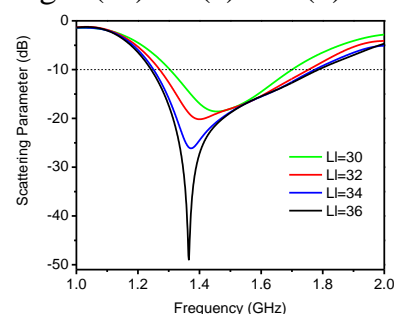

(b)
Figure 3: Effect of horizontal L-length $\left(L_{l}\right)$ on (a) AR (b) $S_{11}$

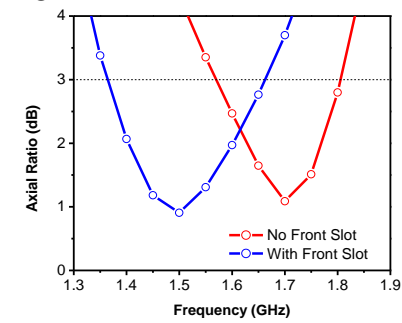

(a)

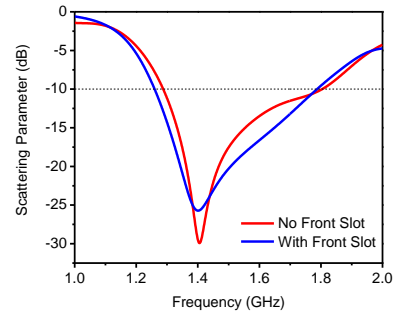

(b)
Figure 4: Effect of the supplementary slot on (a) AR (b) $S_{11}$

Nonetheless, the horizontal L-shaped feed-line length $\left(L_{l}\right)$ was found to be the parameter that had substantial effect on both impedance matching and CP bandwidth as can be seen from Fig. 3. As for the ARBW, it has been shown to be controlled by the supplementary slot next to the upper half of the vertical cross-slot, separated by a distance $s$. The significant effect can be seen in Fig. 4 as the ARBW was shifted without significant impact on the impedance matching. Fig. 4(b) shows that the optimised design had a simulated RLBW of 1.26 to $1.79 \mathrm{GHz}$ (i.e. $34.8 \%$ ), whereas Fig. 5 shows that the simulated ARBW ranges from 1.36 to $1.66 \mathrm{GHz}$ (i.e. $20 \%$ ) occupying $58 \%$ of the total RLBW. With a bidirectional radiation property, the antenna has a maximum gain ranging from $3.1-3.7 \mathrm{dBi}$ with a total efficiency of more than $90 \%$ across the operating band.

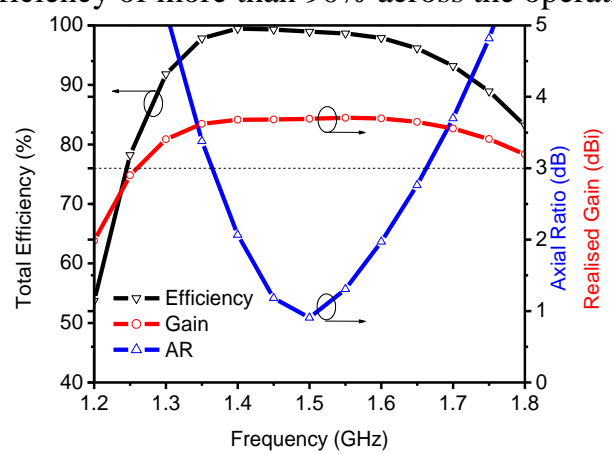

Figure 5: Simulated Total Efficiency, AR and Gain

\section{Simulated and Measured Results}

Fig. 6 displays the simulated surface current distributions of the proposed antenna at the centre frequency of $1.5 \mathrm{GHz}$ to illustrate the left-hand CP (LHCP) operation, having the major current distribution of the same direction at the phases of $45^{\circ}$ and $225^{\circ}$, but opposite to that obtained at $135^{\circ}$ and $315^{\circ}$.

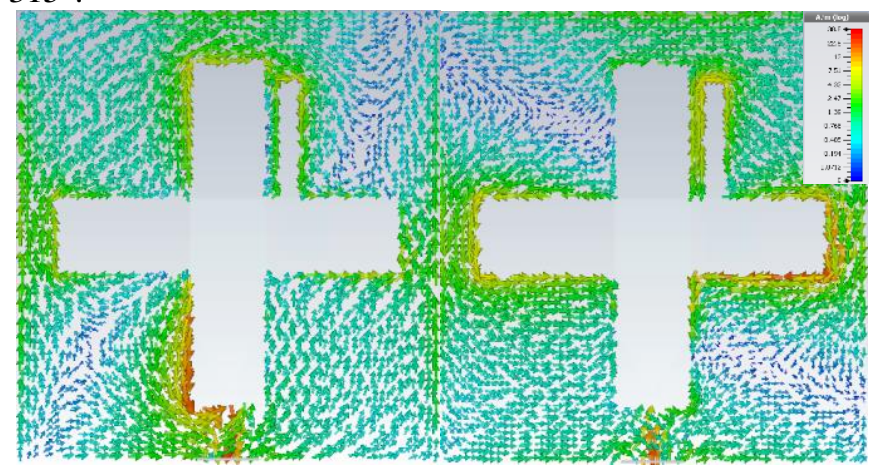

(a) $\omega \mathrm{t}=45^{\circ}$

(b) $\omega \mathrm{t}=135^{\circ}$

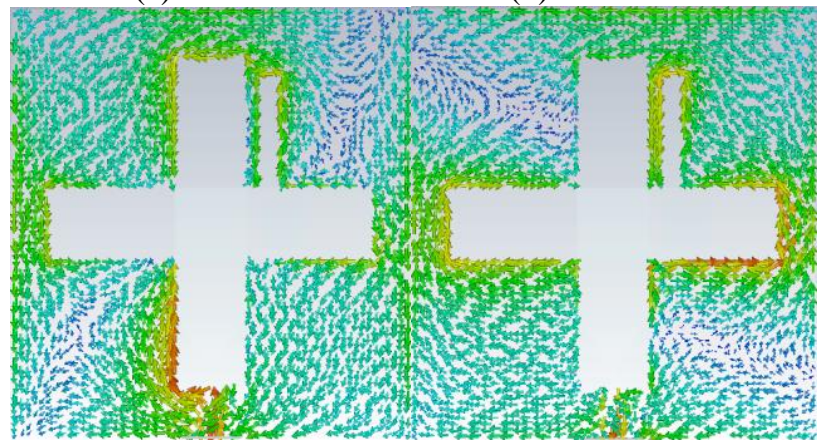

(c) $\omega t=225^{\circ}$

(d) $\omega \mathrm{t}=315^{\circ}$

Figure 6: Surface current distribution at $1.5 \mathrm{GHz}$ at different phase angles 
A prototype of the proposed slot antenna was fabricated and measured. Fig. 7 displays the simulated and measured scattering parameter with an inset of the prototype antenna which was measured by an Agilent Network Analyser N5230A. A good agreement with a slight shift is observed for the measured RLBW to be from 1.3 to $1.83 \mathrm{GHz}$ (i.e. $34 \%$ ).

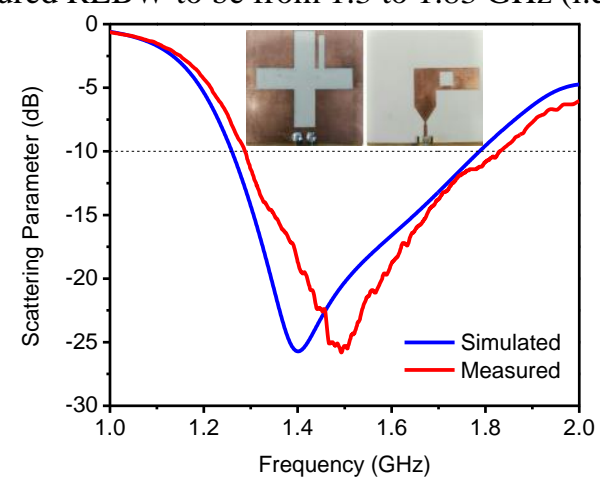

Figure 7: Measured $S_{11}$ in comparison with simulation

The calculated AR result is compared to the simulated one in Fig. 8. Calculations of AR were undertaken using the equations in [9] with the readings of both the amplitude and phase for the horizontal and vertical planes $\left(\mathrm{H}_{\mathrm{A}}, \mathrm{V}_{\mathrm{A}}\right.$ and $\mathrm{H}_{\mathrm{P}}$, $\left.\mathrm{V}_{\mathrm{P}}\right)$ :

$$
\begin{gathered}
E_{L H C P}=1 / \sqrt{2}\left\{\begin{array}{l}
{\left[H_{A} \cos \left(H_{P}\right)+V_{A} \sin \left(V_{P}\right)\right]} \\
+j\left[H_{A} \cos \left(H_{P}\right)-V_{A} \sin \left(V_{P}\right)\right]
\end{array}\right\} . \\
E_{R H C P}=1 / \sqrt{2}\left\{\begin{array}{l}
{\left[H_{A} \cos \left(H_{P}\right)-V_{A} \sin \left(V_{P}\right)\right]} \\
\left.+j\left[H_{A} \cos \left(H_{P}\right)+V_{A} \sin \left(V_{P}\right)\right]\right\}
\end{array}\right\} . \\
A R=\frac{\left|E_{R H C P}\right|+\left|E_{L H C P}\right|}{\left|E_{R H C P}\right|-\left|E_{L H C P}\right|} \\
A R_{d B}=20 \times \log _{10}(A R)
\end{gathered}
$$

The result showed reasonably good agreements at most of the frequencies between simulation and measurement, recording a $13 \%$ 3-dB ARBW (from 1.4 to $1.6 \mathrm{GHz}$ ). This is narrower than the simulated, which can be attributed to the undesired effect of measuring cables and feeding port on the radiation. Fig. 9 shows the measured radiation patterns in comparison to the simulated results at the start, centre, and end frequencies: $1.3,1.5$, and $1.8 \mathrm{GHz}$. Only half of the polar plot is shown because the radiation to the opposite side was significantly distorted by the rotation joint which partially blocks the backward radiation in the used testing configuration. Finally, Fig.10 illustrates both the simulated LHCP and right hand CP (RHCP) patterns at the centre frequency of $1.5 \mathrm{GHz}$ for the $\mathrm{x}$ $\mathrm{z}$ plane, indicating the $\mathrm{CP}$ radiation at the boresight direction.

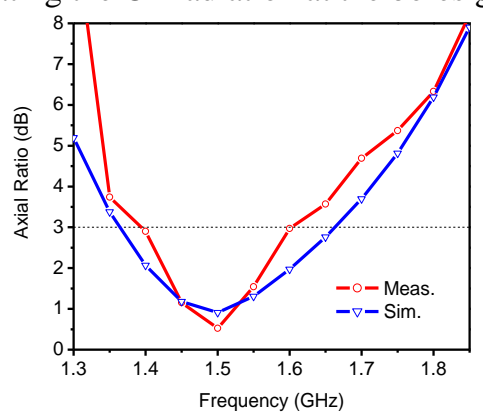

Figure 8: Measured AR in comparison with simulation

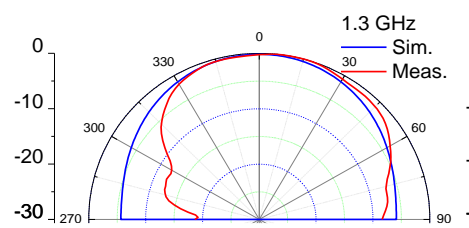

(a)

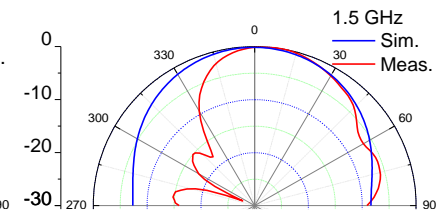

(b)

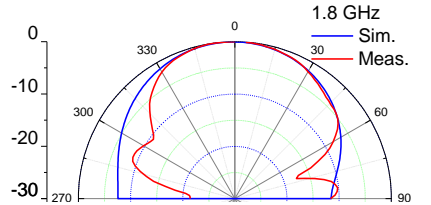

(c)

Figure 9: Simulated versus measured radiation patterns at $\mathrm{x}-\mathrm{z}$ plane (a) $1.3 \mathrm{GHz}$ (b) $1.5 \mathrm{GHz}$ (c) $1.8 \mathrm{GHz}$

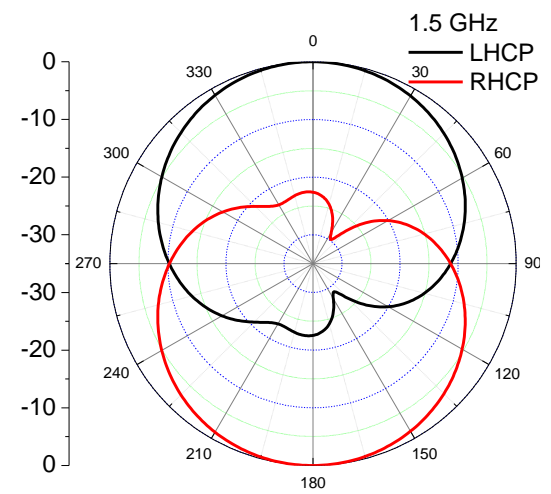

Figure 10: Simulated LHCP and RHCP patterns at the x-z plane of $1.5 \mathrm{GHz}$

\section{Conclusion}

A prototype LHCP slot antenna with an L-shaped strip feedline has been designed, fabricated, and measured. The wideband $\mathrm{CP}$ operation is achieved by properly positioning the L-shaped metal strip and adjusting its lengths underneath the cross-slot antenna. Enhanced impedance matching is accomplished by optimising the taper to the L-shaped strip. The ARBW has been controlled mainly by the supplementary slot next to the upper vertical cross-slot. Good agreement between the simulated and measured $\mathrm{S}_{11}$ has been attained exhibiting a 34\% (34.8\%) measured (simulated) RLBW. The measured (simulated) ARBW is $13 \%$ (20\%). A good agreement in the bi-directional radiation pattern at the centre frequency of $1.5 \mathrm{GHz}$ has been achieved. It is worth mentioning that by adding a reflector underneath this design at a distance of around quarter wavelength $[\sim 50 \mathrm{~mm}]$, a unidirectional pattern with more than doubled maximum gain can be obtained. However, this has an adverse impact on the ARBW, even though the RLBW remained nearly affected.

\section{Acknowledgements}

MGA would like to thank the scholarship offered by Modern Sciences \& Arts University (Egypt) and the University of Greenwich (UK) for the funded resources. Thanks go to Dr Yun $\mathrm{Wu}$ and Amira El-Toukhy for their assist in the measurements. 


\section{References}

[1] S. S. Gao, Q. Luo, and F. Zhu, Circularly Polarized Antennas. John Wiley \& Sons, 2014.

[2] X. Chen, C. G. Parini, B. Collins, Y. Yao, and M. Ur Rehman, Antennas for Global Navigation Satellite Systems. John Wiley \& Sons, 2012.

[3] T. Fujimoto and T. Yoshida, "A printed Inverted-F Antenna for Circular Polarization," IEEE Antennas Propag. Soc. Int. Symp. APSURSI Proc., pp. 21712172, 2016.

[4] F. Chang, K. Wong, and T.-W. Chiou, "Low-Cost Broadband Circularly Polarized Patch Antenna," IEEE Trans. Antennas Propag., vol. 51, no. 10, pp. 3006-3009, 2003.

[5] M. Nosrati and N. Tavassolian, "Miniaturized Circularly Polarized Square Slot Antenna with Enhanced Axial-Ratio Bandwidth Using an Antipodal Y-strip," IEEE Antennas Wirel. Propag. Lett., vol. PP, no. 99, pp. 1-4, 2016.

[6] P. Mousavi, B. Miners, and O. Basir, "Wideband LShaped Circular Polarized Monopole Slot Antenna," IEEE Antennas Wirel. Propag. Lett., vol. 9, pp. 822$825,2010$.

[7] H. Tang, K. Wang, R. Wu, and C. Yu, "Compact Broadband CP Monopole Antenna With Tilted Branch," Electron. Lett., vol. 52, no. 21, pp. 17391740, 2016.

[8] K. Ding, C. Gao, T. Yu, and D. Qu, "Broadband CShaped Circularly Polarized Monopole Antenna," IEEE Trans. Antennas Propag., vol. 63, no. 2, pp. 785-790, 2015.

[9] B. Y. Toh, R. Cahill, and V. F. Fusco, "Understanding and Measuring Circular Polarization," IEEE Trans. Educ., vol. 46, no. 3, pp. 313-318, 2003. 